\title{
Blood pressure response to dietary modification is associated with use of anti-hypertensive therapy
}

\author{
C. E. Huggins, A. Worsley, C. Margerison, M. K. Jorna and C. A. Nowson \\ Centre for Physical Activity and Nutrition Research, School of Exercise and Nutrition Sciences, Deakin University, \\ Burwood, Victoria 3125, Australia
}

It has previously been reported that two diets lower blood pressure (BP): one based on the dietary approaches to stop hypertension (DASH)-type diet, which is high in fruits, vegetables and low-fat dairy foods; a second diet that is low in Na and high in K (LNAHK) ${ }^{(1)}$. Moreover, a greater BP reduction was demonstrated to be achieved after consuming the LNAHK compared with the DASH-type diet (DASH). Whether the BP response to either the DASH (OD) or the LNAHK diets is affected by anti-hypertensive medication is not known. The aims of the present study were to determine: (1) if the BP response to DASH and LNAHK differs between those taking antihypertensive medications and those not taking anti-hypertensive medication; (2) for those who were taking anti-hypertensive medication, if the BP response differs by anti-hypertensive medication class. Ninety-four subjects, which included twenty-four men and eighteen women taking anti-hypertensive therapy, completed a 12-week study in which, following a 2-week control diet (CD), all subjects followed two dietary regimens (for 4 weeks each) in random order, i.e. DASH, plus either a LNAHK or high-dairy diet with a second 2-week CD period between diets. Home BP was measured daily for the last 2 weeks in each phase ${ }^{(1)}$. Anti-hypertensive medication was classified as either renin-angiotensin system blockade (ACE/AT1) or other anti hypertensive therapies (Ca-channel blockers and $\beta$-adrenergic blockers). In subjects who were taking anti-hypertensive medication, the OD diet did not significantly lower systolic BP (SBP; mean difference -1.1 (SE 0.9) $\mathrm{mmHg} ; P>0.05$ ) relative to the preceding CD phase. The greatest fall in SBP was detected in subjects on the LNAHK diet who were taking anti-hypertensive medication (mean difference -6.2 (SE 1.3 ) $\mathrm{mmHg}$ ) and this fall was markedly greater than that of dietmatched non-medication users $(P=0.036$; ANOVA; Figure $)$.

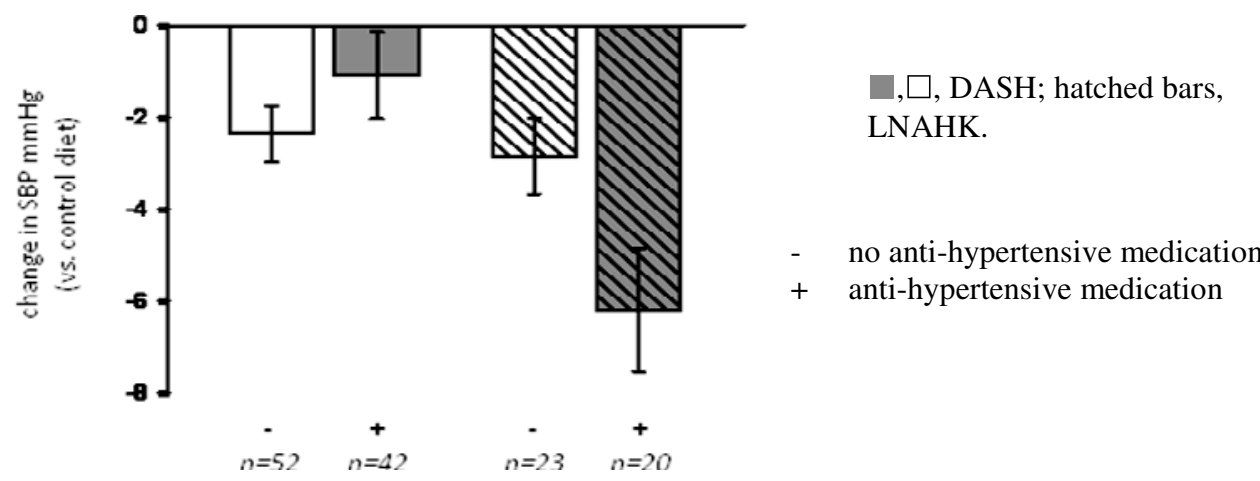

Figure. Change in SBP, by diet type, split by anti-hypertensive medication use.

Compared with the CD, DASH reduced SBP in subjects receiving ACE/AT1 (mean difference $-4.2(\mathrm{SE} 0.2) \mathrm{mmHg}, n 15 ; P<0.01)$ but not in those taking other anti-hypertensive therapies (mean difference +0.6 ( $\mathrm{SE} 1.2) \mathrm{mmHg}, n 27 ; P>0.05$ ). There was a significant fall in BP in those consuming LNAHK diet and taking ACE/AT1 (mean difference; SBP - 9.5 (SE 2.4) mmHg; diastolic BP (DBP) -4.1 (SE 1.3 ) $\mathrm{mmHg}, n 7 ; P<0.01$ and $P<0.05$ respectively) compared with the $\mathrm{CD}$. For those taking other anti-hypertensive therapies, the LNAHK significantly lowered SBP (mean difference -4.4 (SE 1.4 ) $\mathrm{mmHg}, n 13 ; P<0.01$ ) but not DBP (mean difference -2.5 (SE 1.3) $\mathrm{mmHg} ; P>0.05)$ compared with the CD. Implementation of a LNAHK diet may be a useful adjunct treatment in reducing BP, particularly in those taking ACE/AT1 anti-hypertensive medication.

1. Nowson CA, Worsley A, Margerison C et al. (2004) J Nutr 134, 2322-2329. 